\title{
Peptidomimetic 1-Benzyl-5-methyl-4-(n- octylamino)pyrimidin-2(1H)-one Showed Cardioprotection Effect in a Myocardial Ischemia (MI) Mouse Model +
}

\author{
Lena Trifonov ${ }^{1}$, Vadim Nudelman ${ }^{2}$, Michael Zhenin ${ }^{1}$, Guy Cohen ${ }^{3}$, Krzysztof Jozwiak ${ }^{4}$, \\ Edward Korshin ${ }^{1}$, Hanoch Senderowitz ${ }^{1}$, Asher Shainberg ${ }^{5}$, Edith Hochhauser ${ }^{2}$ and \\ Arie Gruzman 1,* \\ 1 Department of Chemistry, Faculty of Exact Sciences, Bar-Ilan University, Ramat Gan 5290002, Israel \\ 2 Cardiac Research Laboratory, Felsenstein Research Center, Rabin Medical Center, Sackler Faculty of \\ Medicine, Tel Aviv University, Petah Tikva 4910002, Israel \\ 3 The Skin Research Institute, The Dead-Sea and Arava Science Center, Masada 86910, Israel \\ 4 Department of Biopharmacy, Medical University of Lublin, 20-093 Lublin, Poland \\ 5 Faculty of Life Sciences, Bar-Ilan University, Ramat Gan 5290002, Israel \\ * Correspondence: gruzmaa@biu.ac.il \\ + Presented at the 2nd Molecules Medicinal Chemistry Symposium (MMCS): Facing Novel Challenges in \\ Drug Discovery, Barcelona, Spain, 15-17 May 2019.
}

Published: 14 August 2019

Keywords: peptidomimetics; cardioprotection; in silico modeling

TLR4, a member of the toll-like receptors (TLRs) family, serves as a pattern recognition receptor in the innate immune response to different microbial pathogens. TLR4 also might be activated by endogenous factors produced by different stress stimuli or cell damage and regulates the inflammatory reaction to ischemic injury in the heart tissues. TRIF-related adaptor molecule (TRAM) serves as an adapter that recruits the Toll/interleukin 1 receptor (TIR) domain-containing adapterinducing IFN- $\beta$ (TRIF) to activate TLR4, following TRIF-dependent cytokine gene transcription. Based on a known TRAM-derived decoy, a nine-amino acid peptide [1], which corresponds to sequences from the TIR domain, the minimal effective sequence resultant in tetrapeptide was evaluated in cardiomyocytes. Then, a simplified peptidomimetic framework was designed, and ten peptidomimetics of this type were synthesized. One of them, namely 1-benzyl-5-methyl-4- $(n-$ octylamino)pyrimidin-2(1H)-one (1), exhibited high potency and efficacy in vitro. Obtained in vitro data and in silico analysis provided evidences for a direct interaction of $\mathbf{1}$ with the TLR4 complex. Being administered in mice with MI, peptidomimetic $\mathbf{1}$ was able to block the pathophysiological manifestation of MI, resulting in normalization of $\mathrm{CK}, \mathrm{LDH}$, and troponin blood levels, restoration of the concomitant tissue damage, and a 100\% survival rate. Inhibition of TLR4-mediated inflammation in post-ischemic myocardium might be used as a therapeutic approach for developing novel cardioprotective drugs [2].

\section{References}

1. Piao, W.; Vogel, S.N.; Toshchakov, V.Y. Inhibition of TLR4 signaling by TRAM-derived decoy peptides in vitro and in vivo. J. Immunol. 2013, 190, 2263-2272. 
2. Trifonov, E.; Nudelman, V.; Zhenin, M.; Matsree, E.; Afri, M.; Schmerling, B.; Cohen, G.; Jozwiak, K.; Weitman, M.; Korshin, E.; et al. A peptidomimetic: 1-benzyl-5-methyl-4-(octylamino)pyrimidin-2(1H)-one designed based on the structure of in TLR4 related TRIF-related adaptor molecule (TRAM)-Decoy peptide, showed cardio protective effect in myocardial ischemia mouse model. J. Med. Chem. 2018, 61, 11309-11326.

(C) 2019 by the authors. Licensee MDPI, Basel, Switzerland. This article is an open access article distributed under the terms and conditions of the Creative Commons Attribution (CC BY) license (http://creativecommons.org/licenses/by/4.0/). 